\title{
CONOCIMIENTO DE ENFERMERIA SOBRE EL CONSUMO DE SUSTANCIAS PSICOACTIVAS Y SU PREVENCIÓN
}

\section{NURSING KNOWLEDGE ON THE USE OF PSYCHOACTIVE SUBSTANCES AND THEIR PREVENTION}

\author{
TITULO CORTO: CONOCIMIENTO DE ENFERMERÍA SOBRE EL CONSUMO DE \\ SUSTANCIAS PSICOACTIVAS
}

\section{Doriam Camacho-Rodríguez ${ }^{1}$ y Gisela González-Ruíz ${ }^{2}$}

Recibido en marzo 03 de 2015

Aceptado en abril 29 de 2015

\section{RESUMEN}

Identificar el nivel de conocimientos de los profesionales de Enfermería de una institución de salud de primer nivel de atención sobre el consumo sustancias psicoactivas y su prevención. Estudio descriptivo de corte transversal en el que participaron los profesionales de Enfermería de una Institución Prestadora de Servicios de salud pública de primer nivel de atención de Santa Marta, Colombia. Como resultado se encontró que el nivel de conocimientos sobre generalidades y prevención es medio $(\overline{\mathrm{x}}=3,8)$ y $(\overline{\mathrm{x}}=3,3)$ respectivamente, predominando el conocimiento sobre sustancias legales $(\overline{\mathrm{X}}=4,0)$ y factores de riesgo y protectores $(\overline{\mathrm{x}}=4,9)$. Se evidencia un nivel de conocimientos medio sobre el tema de prevención de consumo de sustancias psicoactivas.

Palabras clave: Conocimiento; trastornos; Enfermería de atención primaria; promoción de la salud; prevención de enfermedades (Fuente: DeCS).

\section{ABSTRACT}

Identify the level of knowledge of nursing professionals in a primary health Institution about consumption of psychoactive substances and prevention. Cross sectional study involving nursing professionals in an Institution, provider of public health primary care in Santa Marta, Colombia. As result, it was found the level of knowledge about prevention is generalities and a half $(\overline{\mathrm{X}}=3,8)$ and $(\overline{\mathrm{X}}=3,3)$ respectively, prevailing knowledge about legal substances $(\overline{\mathrm{x}}=4,0)$ and risk and protective factors $(\overline{\mathrm{X}}=4,9)$. Evidence means a level of knowledge about prevention of substance use.

Keywords: Knowledge; disorders; primary care nursing; health promotion; disease prevention (Fuente: Mesh).

\footnotetext{
1. Enfermera, Magister en gestión de la calidad, prevención y medio ambiente. Coordinadora de Investigación Facultad de Enfermería, Universidad Cooperativa de Colombia sede Bucaramanga - Colombia. Correo electrónico: doriam.camacho@campusucc.edu.co; doricama@yahoo.es

2. Enfermera, Magister en Ciencias Básicas biomédicas. Profesora Investigadora Facultad de Enfermería, Universidad Cooperativa de Colombia, sede Santa Marta Colombia. Correo electrónico: gisela.1060@gmail.com
} 


\section{INTRODUCCIÓN}

A ctualmente el consumo de tabaco es una de las principales problemáticas a nivel mundial, ya que está relacionado con el desarrollo de enfermedades mortales ${ }^{1}$. La Organización Mundial de la Salud lo catalogó como un problema de salud pública en 2001 y, en 2004 estimó en 4,9 millones las muertes anuales relacionadas con el consumo de tabaco, datos confirmados y superados por el Informe Mundial de Drogas de 2008, de la Oficina de las Naciones Unidas contra la Droga y el Delito².

En Colombia, el primer lugar de consumo de sustancias legales en escolares lo ocupan las sustancias psicoactivas legales, es decir, alcohol y tabaco. En relación con el consumo de sustancias ilegales, el 12,1\% de los estudiantes declaró haber consumido una de estas sustancias al menos una vez en su vida, siendo mayor el consumo en hombres (14\%) que en mujeres $(10,3 \%)^{3}$. Esta tendencia es similar en el resto de la población, encontrándose en el 2013 un leve descenso en el consumo de tabaco y un incremento en el consumo de cocaína ${ }^{4}$.

Teniendo en cuenta esta problemática, la Comisión Interamericana para el Control del Abuso de Drogas (CICAD), organismo de la Organización de Estados Americanos (OEA) luego del análisis y evaluación de las estrategias utilizadas para la reducción de la demanda de drogas ilícitas, decide a partir del año 1996 implementar una estrategia de formación de enfermeros en el área de reducción de sustancias psicoactivas, teniendo en cuenta su amplia participación en los programas de promoción de la salud y prevención de la enfermedad y su capacidad para trabajar en equipos interdisciplinarios y entrar en contacto con la comunidad 5 .

La política nacional para la reducción del consumo de sustancias psicoactivas (SPA) y su impacto establece que para reducir la incidencia del consumo de SPA en el país, es necesario avanzar hacia la prevención, es decir, evitar/prevenir el inicio y uso indebido de sustancias psicoactivas en cualquier momento del ciclo vital ${ }^{6}$, lo cual exige que se diseñen e implementen programas de prevención con mayor impacto ${ }^{7}$. El Plan estratégico Regional de la Contribución de Enfermería en el área de Reducción de la demanda de drogas en América Latina, plantea que el profesional de enfermería es un referente indispensable como miembro del equipo de salud para la discusión de planes y políticas estratégicas en la reducción de la demanda de drogas, dada su potencialidad en las actividades de promoción, prevención e integración social, y el desarrollo en modelos de liderazgo para actuar a nivel local ${ }^{8}$.

Este estudio sirve de punto de partida para identificar el nivel de conocimientos de los profesionales de Enfermería de primer nivel de atención sobre generalidades del consumo sustancias psicoactivas y su prevención, y de esa manera poder brindar una guía respecto a las temáticas en las que requiere actualización el personal de Enfermería responsable de la atención en programas de promoción de la salud y prevención de la enfermedad, así como reforzar en la formación de profesionales de enfermería para actuar frente a la prevención del consumo de sustancias psicoactivas en la población del área de influencia.

\section{MATERIALES Y METODOS}

El estudio es descriptivo de corte transversal. La población objeto fueron los profesionales de Enfermería de una Institución Prestadora de Servicios de salud pública, de primer nivel de atención de Santa Marta, Colombia, y el muestreo fue censal $(\mathrm{N}=15)$.

La información se recolectó a partir de dos cuestionarios elaborados por Sánchez ${ }^{9}$. El primer instrumento mide el nivel de conocimientos sobre el consumo de sustancias psicoactivas, y el segundo mide el nivel de conocimientos sobre programas de prevención de dicho consumo. Los dos instrumentos utilizan una escala tipo Likert y la medición se realiza según el resultado de la media aritmética, así: nivel de conocimientos alto (entre 4 y 5), medio (entre 3 y 3,99 ) y bajo (entre 0 y 2,99 ).

Los cuestionarios fueron auto-administrados en el lugar de trabajo de los participantes en la investigación.

El análisis de los datos se realizó a través del paquete estadístico SPSS versión 20.

Siguiendo las consideraciones éticas de la Resolución 008430 de 1993, esta investigación se considera de bajo riesgo y los participantes firmaron un consentimiento informado; además se les informó que los resultados de la investigación solo se utilizarían con fines académicos y que se garantizarían los principios de confidencialidad y privacidad.

\section{RESULTADOS}

La media de edad de los participantes es de 35 años, el $100 \%(\mathrm{~N}=15)$ son de género femenino y con nivel 
de formación de pregrado; la mayoría egresaron en los últimos 10 años de la universidad, el $44 \%$ entre los años 2006 y 2011, y el 19\% entre los años 2000 y 2005. Respecto del tipo de institución educativa de la cual egresaron, el $100 \%$ se graduaron de universidades de la costa caribe colombiana, el 53,3\% de universidades del régimen de economía solidaria, $33,3 \%$ de universidades privadas, y el $13,3 \%$ de universidades públicas.

El nivel de conocimientos sobre diversos aspectos relacionados con las sustancias psicoactivas es medio ( $\overline{\mathrm{x}}=3,8$ ), según la escala de valoración; sin embargo, el nivel de conocimientos fue alto en relación con efectos y consecuencias a corto, mediano y largo plazo derivados del consumo $(\overline{\mathrm{x}}=4,1)$, factores de riesgo asociados al consumo $(\overline{\mathrm{x}}=4,1)$ e impacto en rendimiento escolar y relaciones familiares/sociales del consumo $(\overline{\mathrm{X}}=4,0)$.

Se encontró que las sustancias psicoactivas sobre las cuales existe un mayor nivel de conocimientos en la población estudiada fueron el alcohol $(\overline{\mathrm{x}}=4,1)$ y el tabaco $(\overline{\mathrm{x}}$ $=4,0)$, en tanto que la menos conocida es el éxtasis $(\bar{x}$

$=3,6$ ). En relación con las variables, las más conocidas son efectos y consecuencias a corto, mediano y largo plazo del alcohol $(\overline{\mathrm{x}}=4,1)$ y factores de riesgo asociados al consumo $(\overline{\mathrm{x}}=4,1)$, y las de menor conocimiento son forma de presentación $(\overline{\mathrm{x}}=3,3)$ y principales mitos existentes acerca del consumo $(\overline{\mathrm{x}}=3,4)$ (Tabla 1).

Tabla 1. Nivel de conocimientos sobre diversos aspectos relacionados con las sustancias psicoactivas

\begin{tabular}{|l|c|c|c|c|c|c|c|c|}
\hline \multicolumn{1}{|c|}{ Variable } & Tabaco & Alcohol & Marihuana & Cocaína & Éxtasis & Heroína & Total & s \\
\hline Forma de presentación & - & - & 3,3 & 3,3 & 3,1 & 3,3 & 3,3 &, 524 \\
\hline Forma de consumo & - & - & 3,3 & 4,4 & 3,2 & 3,2 & 3,5 & 1,033 \\
\hline $\begin{array}{l}\text { Efectos y consecuencias } \\
\text { a corto, mediano y largo } \\
\text { plazo derivados del } \\
\text { consumo }\end{array}$ & 4,2 & 4,3 & 4,1 & 3,9 & 3,9 & 3,9 & 4,1 &, 516 \\
\hline $\begin{array}{l}\text { Principales mitos } \\
\text { existentes acerca del } \\
\text { consumo }\end{array}$ & 3,5 & 3,6 & 3,4 & 3,3 & 3,3 & 3,3 & 3,4 &, 617 \\
\hline $\begin{array}{l}\text { Conductas de riesgo } \\
\text { asociadas al consumo }\end{array}$ & 4 & 4,2 & 3,9 & 3,7 & 3,6 & 3,8 & 3,9 &, 834 \\
\hline $\begin{array}{l}\text { Impacto en rendimiento } \\
\text { escolar y relaciones } \\
\text { familiares/sociales del } \\
\text { consumo }\end{array}$ & 4 & 4,1 & 4 & 4 & 3,9 & 4 & 4 &, 655 \\
\hline $\begin{array}{l}\text { Factores de riesgo } \\
\text { asociados al consumo }\end{array}$ & 4,1 & 4,1 & 4,1 & 4,1 & 3,9 & 4,1 & 4,1 &, 516 \\
\hline $\begin{array}{l}\text { Factores protectores } \\
\text { asociados al consumo }\end{array}$ & 4,1 & 4,3 & 3,8 & 3,8 & 3,8 & 3,8 & 3,9 &, 834 \\
\hline $\begin{array}{l}\text { Total } \\
\text { f, }\end{array}$ & 4,0 & 4,1 & 3,7 & 3,8 & 3,6 & 3,7 & 3,8 &, 884 \\
\hline
\end{tabular}


Cabe anotar que no se encontró asociación entre el nivel de conocimientos de los profesionales de Enfermería y el año de egreso de la universidad, pero sí se encontró asociación entre edad y nivel de conocimientos sobre forma de presentación de la cocaína $\left(\mathrm{chi}^{2}=34,1\right)$; presentación y forma de consumo de marihuana $\left(\mathrm{chi}^{2}=21,4\right)$ y tabaco $\left(\mathrm{chi}^{2}=25,1\right)$; efectos y consecuencias del consumo del alcohol $\left(\mathrm{shi}^{2}=25,3\right)$, marihuana $\left(\mathrm{shi}^{2}=37,5\right)$, cocaína $\left(\operatorname{chi}^{2}=38,4\right)$, éxtasis $\left(\operatorname{chi}^{2}=38,1\right)$ y heroína $\left(\operatorname{chi}^{2}=38,1\right)$; conductas de riesgo asociadas al consumo de tabaco $\left(\mathrm{chi}^{2}=27\right)$ y alcohol $\left(\mathrm{chi}^{2}=26,2\right)$; impacto en rendimiento escolar y relaciones familiares/sociales del consumo de tabaco $\left(\mathrm{chi}^{2}=23,6\right)$, alcohol $\left(\mathrm{chi}^{2}=24,5\right)$ y marihuana $\left(\mathrm{chi}^{2}=23,6\right)$; factores protectores asociados al consumo de tabaco $\left(\mathrm{chi}^{2}=30\right)$, alcohol $\left(\mathrm{chi}^{2}=30\right)$ y drogas ilícitas $\left(\mathrm{chi}^{2}=30\right)$.

Sobre los conocimientos en materia de programas de prevención del consumo de sustancias psicoactivas, se encontró que el nivel de conocimientos es medio $(\overline{\mathrm{x}}=3,12)$ según la escala de valoración. La variable mejor calificada es “definir prioridades en materia de prevención” $(\overline{\mathrm{X}}=3,54)$. Llama la atención que se encontró un nivel de conocimientos bajo sobre las variables "identificar actividades congruentes con los objetivos operativos" $(\overline{\mathrm{X}}=2,86)$ y "seleccionar indicadores de evaluación” ( $\overline{\mathrm{x}}=2,77)$ (Tabla 2).

Tabla 2. Nivel de conocimientos sobre prevención del consumo de sustancias psicoactivas

\begin{tabular}{|l|c|c|}
\hline Nivel de conocimientos sobre capacidad para: & Media & $\begin{array}{c}\text { Desviación } \\
\text { estándar }\end{array}$ \\
\hline Detectar problemas existentes en la comunidad & 3 &, 756 \\
\hline $\begin{array}{l}\text { Evaluar las necesidades que en materia preventiva tiene la comunidad (conductas } \\
\text { de riesgo, factores asociados al consumo... }\end{array}$ & 3,1 &, 594 \\
\hline $\begin{array}{l}\text { Formular un diagnóstico del problema de consumo de sustancias psicoactivas en la } \\
\text { comunidad }\end{array}$ & 2,9 &, 799 \\
\hline $\begin{array}{l}\text { Justificar ante otras personas (directivas del centro de salud, junta de acción } \\
\text { comunal) la pertinencia de poner en marcha un programa preventivo }\end{array}$ & 3,5 &, 990 \\
\hline Definir prioridades en materia de prevención & 3,54 &, 743 \\
\hline Establecer objetivos operativos & 3,15 &, 640 \\
\hline Identificar actividades congruentes con los objetivos operativos & 2,86 &, 516 \\
\hline Seleccionar indicadores de evaluación & 2,77 &, 676 \\
\hline $\begin{array}{l}\text { Identificar procedimientos para evaluar la forma de implementación y los } \\
\text { resultados obtenidos con el programa }\end{array}$ & 3,33 &, 799 \\
\hline Total & 3,12 &, 594 \\
\hline
\end{tabular}

\section{DISCUSIÓN}

El nivel de conocimientos de los profesionales de Enfermería, de la institución en la cual se realizó la investigación sobre diversos aspectos relacionados con la sustancias psicoactivas fue catalogado como "nivel medio". No obstante, este dato no se puede confrontar con otros estudios, ya que no se encontró evidencia de indagaciones previas en este grupo poblacional y con el mismo tipo de medición. 
Respecto a los factores de riesgo y protectores asociados al consumo de sustancias psicoactivas, el nivel de conocimientos fue catalogado como "nivel alto", lo cual es importante, teniendo en cuenta que el profesional de Enfermería cumple un papel fundamental frente a la identificación de factores de riesgo y el fortalecimiento de los factores protectores, tanto a nivel individual como familiar. Por otra parte, el nivel de conocimientos sobre la temática es importante porque el enfermero sirve como interlocutor entre las necesidades del individuo y la comunidad y los diferentes actores sociales ${ }^{10}$. Al respecto, en este estudio se evidenció un menor nivel de conocimientos en relación con los programas de prevención del consumo de sustancias psicoactivas, en particular en lo relacionado con el establecimiento de objetivos, actividades congruentes con los objetivos e indicadores de evaluación.

Aunque no se encontró relación entre el nivel de conocimientos de los profesionales de Enfermería y el año de egreso de la universidad, cabe anotar que por más de 10 años los contenidos en la temática de drogas se han ido introduciendo en el currículo de formación de más de 100 escuelas y facultades de Enfermería, cuyas cohortes se encuentran hoy en el ambiente laboral aplicando el conocimiento especializado en el tema para problemas específicos de consumo. De esta manera, el proyecto de las Escuelas/Facultades de Enfermería de la SE/CICAD, contribuye de manera sistemática y sostenible a reducir la demanda del consumo de drogas al lanzar al mercado laboral profesionales que cuidan la salud de las personas, y entre su múltiple acción previenen el consumo de drogas y controlan los problemas derivados del consumo de forma directa o indirecta ${ }^{11}$. Según un estudio realizado en Brasil ${ }^{12}$, el $50 \%$ de los estudiantes de Enfermería consideran que la universidad es la mejor instancia para adquirir conocimientos sobre el tema, y particularmente durante el primer año de la carrera, contrario a lo afirmado por Figueroa y Figueroa ${ }^{13}$ quienes recomiendan realizar un abordaje de los estudiantes de los semestres superiores para la correspondiente comprobación de conocimiento sobre las drogas licitas y las drogas ilegales frecuentemente utilizadas. Considerando que la problemática del consumo de drogas sigue en aumento, es indispensable que los enfermeros tengan los conocimientos adecuados para un buen abordaje durante su ejercicio profesional ${ }^{14}$.

Los enfermeros son agentes clave en el trabajo por el cambio y la trasformación de los individuos, familias y comunidades, y cumplen una función esencial en el diseño y ejecución de programas de promoción de la salud y prevención de enfermedades en múltiples campos sociales ${ }^{15}$, y para ello se requiere que los estudiantes lleguen al final de su formación con los conocimientos necesarios para su actuación profesional ${ }^{14}$. El profesional de enfermería puede actuar de forma activa en la prevención del consumo de sustancias psicoactivas, a través de la detección de factores de riesgo y la promoción de factores protectores, aprovechando la oportunidad de interactuar con el individuo y la familia durante la consulta de los programas de protección específica y detección temprana.

A manera de conclusiones, el nivel de conocimientos sobre generalidades y prevención del consumo de sustancias psicoactivas es medio en la población objeto de estudio, lo cual amerita un mejoramiento en los procesos de formación en esta temática, para brindarle al profesional las herramientas cognitivas necesarias para la implementación de programas, estrategias y acciones conducentes a la reducción del consumo de estas sustancias.

Es necesario fomentar la investigación en este grupo poblacional, teniendo en cuenta que no hay antecedentes que permitan contrastar los hallazgos, contrario a lo que sucede con otros miembros del equipo de salud. De hecho, en un estudio realizado con un grupo de docentes y estudiantes del área de la salud de Bolivia, encontraron que existe un desconocimiento sobre las generalidades de las drogas, tanto en profesores como en estudiantes y residentes. De igual forma encontraron que el conocimiento sobre las drogas de uso lícito como son el alcohol y el tabaco tienen un valor positivo muy alto ( $95 \%$ y $87 \%$, respectivamente), seguidos muy cerca por la marihuana con el $83,3 \%$ de las respuestas, similar a lo encontrado en este estudio, donde las sustancias sobre las cuales existe un mayor conocimiento son las legales, alcohol y tabaco ${ }^{16}$; sin embargo, al indagar con los estudiantes de Enfermería, se encontraron deficiencias en relación con la clasificación y los efectos de las sustancias psicoactivas $^{17}$.

Se recomienda a las universidades e instituciones de salud de primer nivel de atención, desarrollar programas de capacitación a los profesionales de Enfermería con el fin de fortalecer las acciones de prevención del consumo de sustancias psicoactivas en cada una de las etapas del ciclo vital, a través del desarrollo de los programas de promoción de la salud y prevención de la enfermedad, 
establecidos en la Resolución 412 de 2000, por la cual se adoptan las normas técnicas y guías de atención para el desarrollo de las acciones de protección específica y detección temprana ${ }^{18}$.

\section{REFERENCIAS BIBLIOGRAFICAS}

1. Gutiérrez G, Londoño E, Calderón Dimaté L, Devia Correa L, González Páez JP. Consumo de tabaco en estudiantes de una Universidad pública del Quindío. Psicogente. 2012; 15 (28):360-370.

2. Prieto C. La escuela y la prevención de drogas: El profesor como un factor de protección en los procesos de prevención del consumo de drogas en la escuela primaria. Cult. Drog. 2009; 14 (16): 61 - 75.

3. Colombia. Ministerio de Justicia y del Derecho, Ministerio de Educación y Ministerio de Protección social. Estudio nacional de consumo de sustancia psicoactivas en Población escolar, 2011.

4. Colombia. Ministerio de Justicia y del DerechoObservatorio de Drogas de Colombia y Ministerio de Salud y Protección Social. Estudio nacional de consumo de sustancias psicoactivas en Colombia -2013. Informe final, 2013.

5. Medina N. REDNACER: Una experiencia para compartir. Bogotá: Editorial Universidad Nacional de Colombia; 2007.

6. Colombia. Ministerio de protección social. Dirección general de salud pública. Política nacional para la reducción del consumo de sustancias psicoactivas y su impacto, 2007.

7. Medina O, Rubio LA. Consumo de sustancias psicoactivas (SPA) en adolescentes farmacodependientes de una fundación de rehabilitación colombiana. Estudio descriptivo. Rev. Colomb. Psiquiat. 2012; 41 (3): 550-561.

8. Comisión Interamericana para el Control del Abuso de Drogas. Plan estratégico Regional de la Contribución de Enfermería en el área de Reducción de la demanda de drogas en América latina. Washington: Organización de Estados Americanos, 2003.

9. Sánchez L. Instrumentos para la evaluación de los programas incluidos en la cartera de servicios en materia de prevención de drogodependencias.
Galicia: Editorial La Coruña: Xunta de Galicia. Servicio de drogodependencia y trastornos adictivos; 2011 [consultado 2014-12-12]. Disponible en http:// www.sergas.es/gal/DocumentacionTecnica/docs/ SaudePublica/adicciones/Doc_Tco_INSTRUMENTOS_ EVALUACION_PREV.pdf

10. Wright G, Luis M, Pillon S, Pires D, Spricio J, Chaves M et al. A Contribuição da Profissão de Enfermagem na Implementação do Plano Nacional Anti-Drogas no Brasil: Uma Proposta em Construção", en: Informe Técnico de la Comisión Interamericana para el Control del Abuso de Drogas/CICAD. Washington: Organización Panamericana de la salud, 2002.

11. Comisión Interamericana para el Control del Abuso de Drogas (CICAD) . La Contribución de las Escuelas de Enfermería en el Área de Reducción de la Demanda de las Drogas en América Latina. Washington: Organización Panamericana de la salud, 2012.

12. Vargas M, Ventura C, Silva E. Conocimientos de estudiantes de Enfermería sobre alcohol y drogas. Rev. Latino-Am. Enfermagem. 2010; 18: 529-34.

13. Figueroa M, Figueroa I. Estado del Conocimiento sobre la Administración de Drogas Lícitas e Ilícitas de los Estudiantes de Enfermería, 2010. [consultado 201408-17]. Disponible en: http://www.portalesmedicos. com/publicaciones/articles/2330/1/Estado-delConocimiento-sobre-la-Administracion-de-DrogasLicitas-e-Ilicitas-de-los-Estudiantes-de-Enfermeria.html

14. Vásquez E, Pillon S. La formación de enfermeras y el fenómeno de las drogas en Colombia: conocimientos, actitudes y creencias. Rev. Latino-Am. Enfermagem. 2005;13 (n.spe): 845-853

15. Abdala M, Fasse D. El currículo de pregrado con el contenido de drogas y la educación continua. En: Comisión Interamericana para el control de abuso de drogas. El currículo de Enfermería con el contenido de drogas para los programas de pregrado y posgrado: la experiencia de la CICAD en América Latina. Washington: Organización Panamericana de la Salud, 2005.

16. Navia M, Farah J, Yaksic N, Magosso A. Conocimiento sobre el fenómeno de las drogas en entre estudiantes y docentes de la Facultad de Medicina Universidad Mayor de San Andrés, La Paz, Bolivia. Rev. Latino-Am. Enfermagem. 2011; 19(n. spe): 722-729 
17. Castrillo M, De la Cruz A, De las salas K, Eguis R, Galves $\mathrm{D}$, Caro S, et al. Conocimientos, actitudes y prácticas relacionados con el consumo de sustancias psicoactivas en estudiantes de enfermería de una universidad del departamento del Atlántico (Colombia). Salud Uninorte. 2012; 28 (2): 322-334.

18. Colombia. Ministerio de Salud. Resolución 412 de 2000 por la cual se establecen las actividades, procedimientos e intervenciones de demanda inducida y obligatorio cumplimiento y se adoptan las normas técnicas y guías de atención para el desarrollo de las acciones de protección específica y detección temprana y la atención de enfermedades de interés en salud pública (Febrero 12 de 2000).

Para citar este artículo: Camacho-Rodríguez D, González-Ruíz G. Conocimiento de enfermería sobre el consumo de sustancias psicoactivas y su prevención. Duazary. 2015 dic; 12 (2): 93 - 99

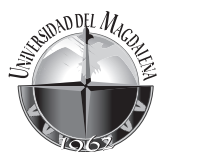

